\title{
Cómo perciben los menores infractores la justicia que se les aplica: un acercamiento desde la justicia procedimental
}

\section{Legitimacy and juvenile justice: perception of juvenile offenders}

\author{
Fátima Pérez Jiménez iD 1 , José Becerra Muñoz iD \\ Araceli Aguilar Conde iD \\ Instituto de Criminología. Universidad de Málaga
}

\begin{abstract}
RESUMEN
Esta investigación pretende conocer en qué medida los menores sancionados perciben el proceso penal como justo, atendiendo a su relación con los distintos operadores jurídicos con los que han tenido contacto. Para ello se ha elaborado un cuestionario ad hoc el cual, tras haber sido sometido a un proceso de validación por expertos, se ha pasado a una muestra de infractores que están cumpliendo una medida sancionadora en la provincia de Málaga. Los resultados dejan ver que los elementos que hacen percibir el proceso penal juvenil como justo pueden no estar suficientemente presentes entre estos jóvenes. Asimismo, las diferencias en la valoración de los distintos profesionales que contactan con ellos parecen ser notables.
\end{abstract}

Palabras clave: justicia juvenil, justicia procedimental, percepción de menores infractores

\section{ABSTRACT}

This research aims to know the perception of sentenced juvenile offenders about the penal process as fair attending to his relation with the different juridical operators who have had contact. A questionnaire has been developed and validated by experts and it has been

\footnotetext{
${ }^{1}$ La correspondencia debe dirigirse a: Fátima Pérez Jiménez. Instituto de Criminología, Edf. I+D. Campus Teatinos, Universidad de Málaga, 29071 Málaga; fatima@uma.es
}

Revista Española de Investigación Criminológica

Artículo 9, Número 16 (2018)

https://doi.org/10.46381/reic.v16i0.171

www.criminologia.net

ISSN: 1696-9219 
answered by a random sample of juvenile offenders who were serving a sentence in the province of Málaga. The results show that the elements that give the perception of the penal juvenile as just process cannot be sufficiently present between these young persons. The differences in the valuation of the different professionals who contact them seem to be important.

Key words: juvenile justice, procedural justice, young offenders' perception

\section{Introducción}

En los últimos años, en el ámbito de los menores y la administración de justicia, se han venido produciendo estudios que tratan de profundizar en el alcance de los presupuestos de la llamada justicia procedimental. El interés primordial de esta línea de trabajo es conocer otros factores que suscitan y promueven el cumplimiento de la ley y la colaboración con la justicia por parte de los menores de edad infractores, más allá del contenido disuasivo de la norma y el castigo previsto en la misma. Se buscan así modos más efectivos para disminuir la comisión de infracciones por parte de los menores (Boonie et al., 2013). En esa línea, este estudio pretende dar un primer paso explorando la percepción de los aludidos presupuestos en menores infractores sancionados y su percepción del papel de los distintos operadores jurídicos, para así poder aportar información a la reflexión en torno a la influencia de un trato justo en la promoción del cumplimiento de las normas entre estos jóvenes.

La investigación realizada revela que una evaluación positiva del sistema de justicia está influida predominantemente por la calidad del trato que la ciudadanía percibe, otorgándosele menos relevancia a los elementos instrumentales del sistema. Tanto Tyler y sus colaboradores (Blader \& Tyler, 2003; Shulfofer, 2011; Sunshine \& Tyler, 2003; Tyler, 1990; Tyler \& Fagan, 2008; Tyler \& Huo, 2002) como otros investigadores (Bradford, 2012; Dai et al., 2011; McCluskey, 2003; entre otros) han ido proponiendo, perfilando y confirmando los distintos aspectos de este fenómeno a lo largo de distintos trabajos. Según este planteamiento, la forma en la que se lleve a cabo el ejercicio concreto de la autoridad se relaciona directamente con la percepción del sujeto de haber sido tratado de una forma justa, influyendo en su comportamiento respetuoso con la ley y la colaboración con los operadores jurídicos. La literatura sostiene que son cuatro los elementos que integran la percepción de un proceso justo: I- el grado en el que

Revista Española de Investigación Criminológica

Artículo 9, Número 16 (2018)

https://doi.org/10.46381/reic.v16i0.171

www.criminologia.net

ISSN: 1696-9219 
se tiene oportunidad de participar y ser escuchado durante el mismo; II- la neutralidad e imparcialidad de los agentes implicados en la toma de decisiones y la transparencia de las mismas; III- el trato educado y respetuoso en las relaciones interpersonales; IV- el grado en el que se considera que las autoridades están actuando con preocupación por la persona y se confía en sus motivaciones (Jonathan-Zamir et al., 2013). De una manera sintética estos elementos se suelen agrupar en dos: la calidad de la toma de decisiones y la calidad del trato recibido (Blader \& Tyler, 2003; Steiner \& Wooldredge, 2015; Tankebe, 2013). Por tanto, si la persona califica de injusto el modo en que los operadores actúan cuando ejercen su autoridad, por ejemplo por no poder participar en las actuaciones o ser objeto de una decisión no ecuánime, o se considera tratada injustamente con ausencia de respeto e interés hacia ella, eso mermará notablemente su intención de cumplir con la ley (Jackson et al., 2012).

En este proceso de investigación acerca de la influencia de la justicia procedimental hay que referirse también a los estudios realizados con sujetos que han tenido contacto con el sistema de justicia penal, iniciados también por Tyler (2001). En estos casos la calidad del trato recibido influye de una manera más notable en la evaluación sobre la justicia, en la aceptación de la sanción impuesta y el cumplimiento de la misma (Tyler, 2003).

Por tanto, las experiencias con la ley y con los operadores jurídicos darán forma a las trayectorias de socialización legal, generando conductas de cumplimiento o de desafío normativo. Esto sugiere que la justicia procedimental produce un efecto directo hacia el cumplimiento normativo y otro indirecto, dando contenido a la percepción sobre la legitimidad (Fagan \& Piquero, 2007) y, en conexión con ello, aquellas experiencias modularán la necesidad de utilizar elementos represivos (Fagan \& Tyler, 2005). Se puede afirmar que la investigación empírica sustenta que la falta de percepción de justicia en el proceso penal está asociada con una escasa apreciación de legitimidad por parte de la ciudadanía (Tyler, 2015).

Los estudios realizados con jóvenes infractores confirman que la calidad de las decisiones y la calidad del trato recibido durante el proceso judicial influyen en la confianza que éstos desarrollan en la autoridad, su atribución de legitimidad y su voluntad 
de cooperación (Reisig et al., 2014). Se trata de factores que incluso juegan un papel importante en la decisión de obedecer la ley en el futuro (entre otras: Braga \& Weisburd, 2012; Kirk \& Matsuda, 2011; McGarrell et al., 2010; Mulvey et al., 2004; Tyler et al., 2014; Walters, 2018) por lo que sería aconsejable que sean tenidas en cuenta a la hora de programar propuestas e intervenciones para la reducción de la delincuencia juvenil.

Otros estudios demuestran cómo las particulares vivencias en la relación con el juez, el fiscal y el abogado afectan significativamente a la confianza en la justicia penal (Greene et al., 2010; Sprott \& Greene, 2008); también cómo los sentimientos de ira o culpa por el delito cometido que sienten los jóvenes en centros de internamiento están influenciados por su percepción de haber sido parte de un proceso justo (Otto \& Dalbert, 2005; Tatar et al., 2012); y, finalmente, cómo la atribución de legitimidad a los funcionarios de prisiones entre los jóvenes reclusos pasa por sentirse objeto de un trato justo y de calidad (Steiner \& Wooldredge, 2015).

Estudios con menores y jóvenes adultos (Agustyn, 2016; Murpy \& Gaylor, 2010; Penner et al., 2014) y estudios longitudinales (Fagan \& Piquero, 2007; Piquero et al., 2005) con menores inmersos en procedimientos penales, concluyen que la edad es un elemento determinante en la percepción de la justicia procedimental y de la legitimidad: sin duda esa percepción es menos estable que en los adultos, pero en los jóvenes de más de 17 años esta experiencia evoluciona en el tiempo y es más sensible a la experiencia personal y vicaria vivida.

El presente estudio pretende explorar en qué medida las propuestas de la justicia procedimental se dan en la práctica de los tribunales penales españoles, en la línea que otros autores han hecho ya en nuestro país (Vázquez Morales \& Fernández-Molina, 2013). Nos centraremos en el ámbito de la justicia juvenil y en las posibles diferencias que cada menor pueda percibir respecto a los distintos operadores jurídicos con los que entra en contacto. Este aspecto no ha sido aún estudiado en nuestro país y aportará una visión desglosada de las experiencias que el menor suma para considerar el proceso penal como justo o injusto.

Al igual que otros autores han tratado de hacer con las particularidades del sistema de justicia juvenil de su país (Birckhead, 2009; Sprott \& Greene, 2008), en este estudio 
se ha buscado tener en cuenta algunas de las singularidades de nuestro proceso penal en la consideración de los elementos que conforman la percepción de un proceso justo. Los tres primeros (participación, neutralidad y trato digno y respetuoso) tienen un contenido acotado y simple de percibir por los jóvenes, sin embargo, el cuarto (preocupación por la persona y confianza) puede aludir a distintas acciones y omisiones que lleven a cabo los operadores jurídicos; de hecho son diversos los aspectos a los que se acude para medir este aspecto de la calidad del trato. Uno de los indicadores utilizados en uno de los estudios mencionados por Tyler (2001) es la comprensión del resultado: "las decisiones del juzgado son entendidas por las personas a las que atañe el proceso"; más recientemente Tyler (2017) también preguntó a jóvenes detenidos por la policía si "te explicaron por qué te habían detenido de un modo comprensible para ti”. Nuestro trabajo presta una atención singular a esta situación debido al "planteamiento híbrido" de la actual regulación española (Bernuz, 2014), en la que "para responder al principio de equidad e individualizar la respuesta judicial, el mismo delito puede acabar con distintas medidas judiciales". Por tanto, se hace necesario comunicar al menor de forma comprensible las razones de las decisiones judiciales en su caso concreto, ya que así se incrementará la aceptación de sus consecuencias.

\section{Método}

\subsection{Objetivos}

El objetivo principal de esta investigación es explorar, en el marco de la justicia procedimental, en qué medida los menores sancionados perciben el proceso penal como justo, atendiendo a su relación con los distintos operadores jurídicos con los que han tenido contacto.

Los objetivos secundarios de la investigación se relacionan con cada uno de los elementos influyentes en la percepción de justicia del proceso: participación, neutralidad, trato digno y respetuoso, y preocupación por la persona. 
1. Examinar la percepción del menor respecto a la oportunidad que se le da durante el procedimiento penal para hablar sobre sus experiencias tras la comisión del delito y de expresar sus sentimientos al respecto.

2. Estudiar la percepción del menor sobre la imparcialidad y objetividad de las decisiones adoptadas durante el procedimiento.

3. Explorar la percepción del menor acerca del trato recibido durante el procedimiento.

4. Conocer si el menor comprende el fundamento de las decisiones y los procedimientos a través de los cuales se toman.

El trabajo que aquí se presenta describe el diseño del instrumento (cuestionario ad hoc auto-administrado), su validación y la realización de un estudio piloto con una muestra de menores sancionados en la provincia de Málaga.

\subsection{El cuestionario y su diseño}

En 1989 Tyler y sus colaboradores propusieron una serie de preguntas para la valoración de los aspectos principales de la justicia procedimental en relación con la experiencia de los infractores respecto a su abogado, al fiscal y al juez. Esta propuesta ha sido utilizada en posteriores investigaciones (Greene et al., 2010; Sprott \& Greene, 2008) con pequeños cambios y ajustes a las realidades procesales específicas, pero singularmente atendiendo a la calidad de las decisiones y a la calidad de trato.

Respecto a la calidad de las decisiones, las preguntas versaban sobre la opinión de los infractores respecto a la honestidad, la imparcialidad, la atención prestada al caso, la neutralidad, el cumplimiento de las normas y la implicación en el caso. En relación a la calidad del trato recibido se les preguntaba si se sentían escuchados comprendiendo lo que decidían los operadores y si habían recibido un trato respetuoso.

Siguiendo este marco y atendiendo también a las características del proceso penal para menores de edad de nuestro país, se han tenido en cuenta en la construcción del instrumento seis dimensiones que cubren ampliamente las facetas que han de ser tenidas en cuenta para la valoración de un proceso justo (

). Las dimensiones 1, 2, 3 y 4 se componen de preguntas relativas a la calidad de la toma de decisiones; las dimensiones 5 y 6 valoran la calidad del trato. Cada una de 
estas dimensiones se analiza en todas las fases del proceso: policial, judicial y de ejecución. Esto da lugar a una multiplicación de los ítems que han de construirse, algo imprescindible para abarcar el gran número de situaciones que finalmente componen la percepción global del menor.

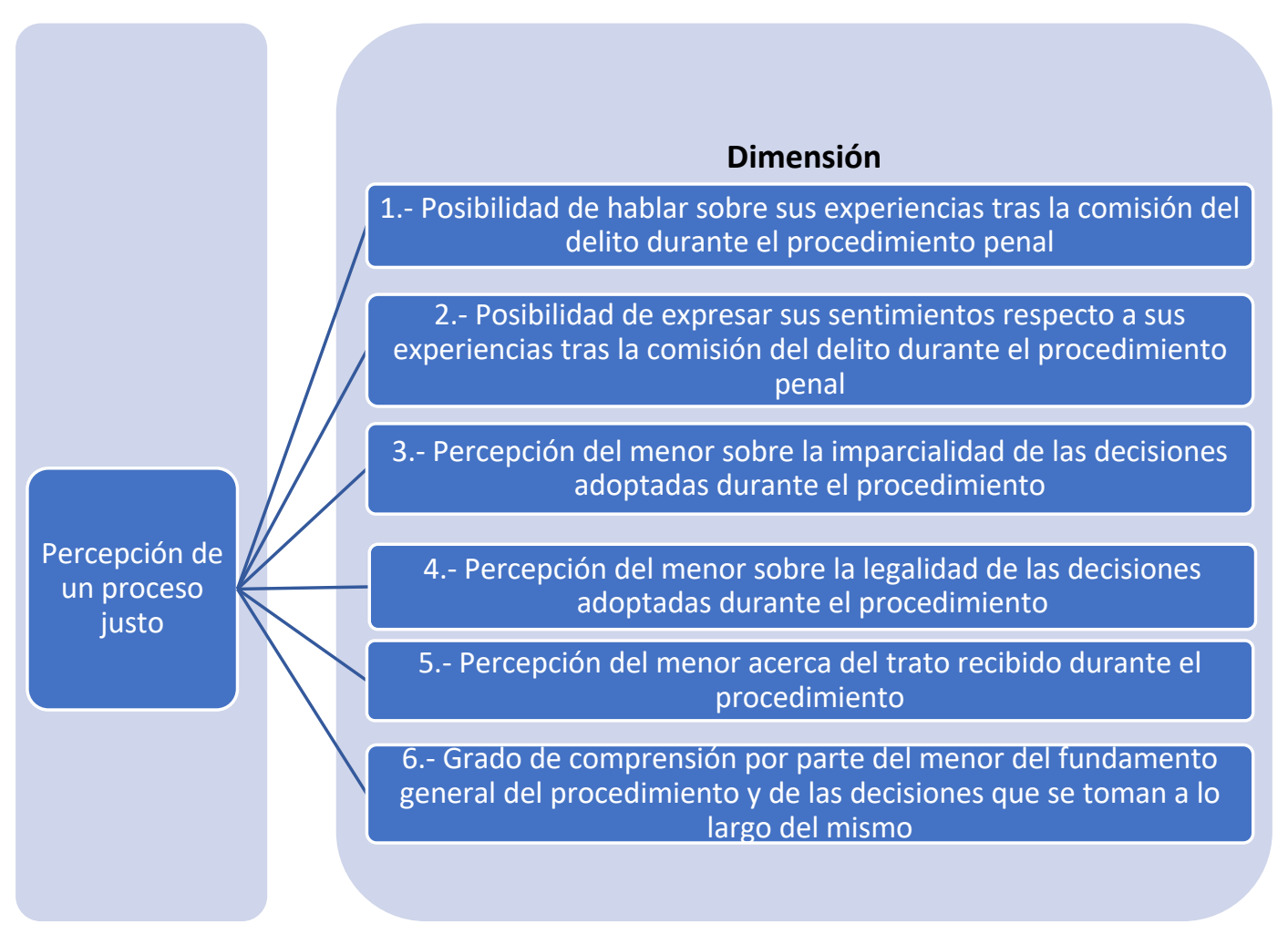

Figura 1. Dimensiones para la valoración de la percepción de un proceso justo

Para el diseño del cuestionario se completaron las siguientes fases (Figura 2).

Revista Española de Investigación Criminológica

Artículo 9, Número 16 (2018)

https://doi.org/10.46381/reic.v16i0.171

www.criminologia.net

ISSN: 1696-9219 


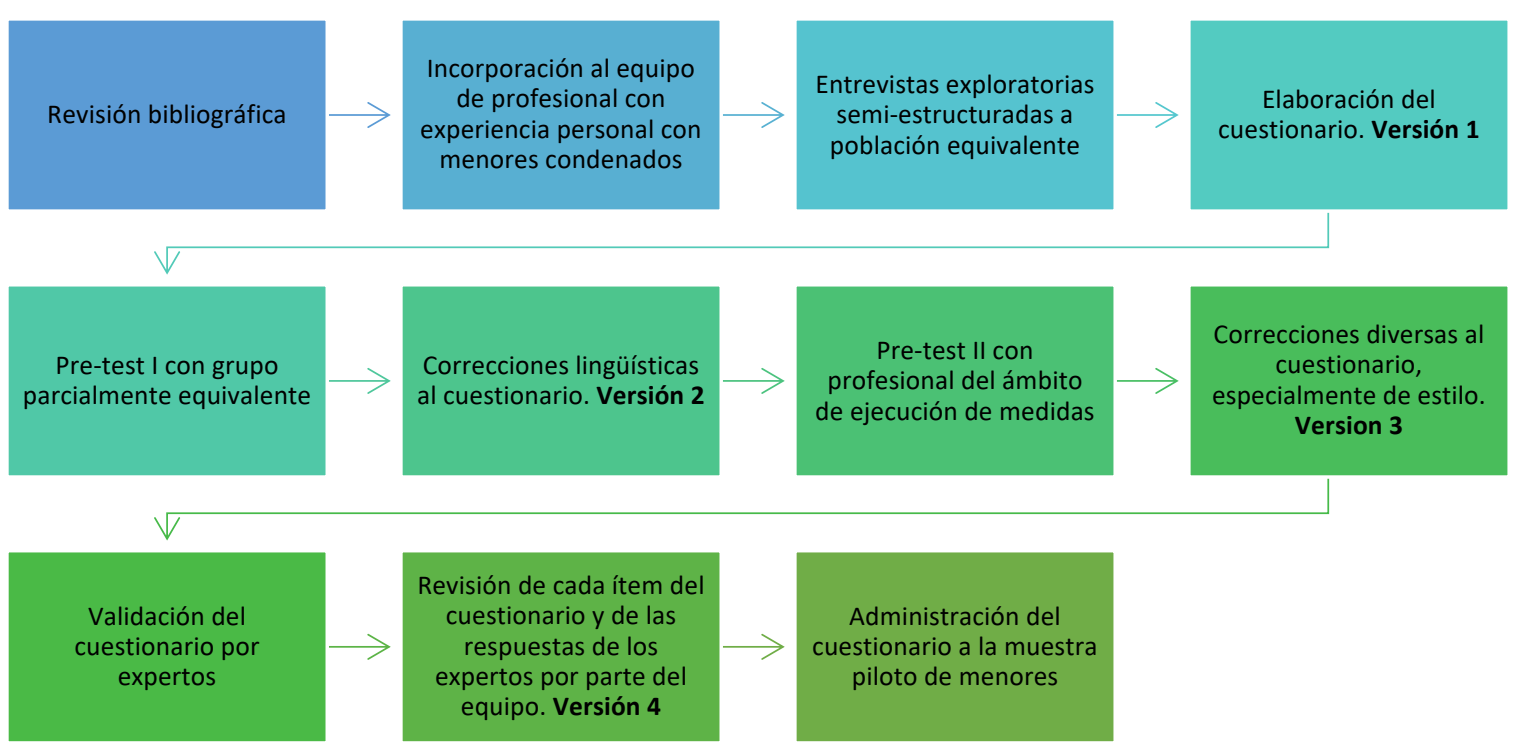

Figura 2. Fases en el diseño del instrumento

Entre las pruebas de validez a las que se ha sometido el cuestionario están la validez de contenido a partir del procedimiento de juicios a expertos y la validez del constructo a partir de un análisis factorial exploratorio. La medida de adecuación muestral KMO tiene un valor de 0,601 lo que muestra que hay variables correlacionadas y la prueba de esfericidad de Barlett es estadísticamente significativa por lo que tiene sentido realizar el análisis factorial exploratorio. Respecto a la prueba de fiabilidad se ha medido el alfa de Cronbach resultando un valor de 0,840. La versión definitiva del instrumento se compone de 55 preguntas.

\subsection{Selección de la muestra y características}

La muestra objeto de estudio está compuesta por un total de 155 sujetos. Se realizó un muestreo aleatorio no probabilístico de conveniencia entre los menores que cumplían alguna medida en la provincia de Málaga. Se contó con un listado facilitado por la Delegación de Justicia de la Junta de Andalucía, que incorporaba los números de identificación personal (NEP) de los menores con expediente activo a 5 de febrero de 2015. Los cuestionarios se remitieron a los centros por correo ordinario junto con las cartas de consentimiento para los padres y tutores de los menores de edad. Los menores 
debían cumplimentar los cuestionarios, introducirlos en un sobre que cerrarían y firmarían, y, finalmente, entregarlos a los profesionales encargados de su administración.

El grupo de menores condenados que ha conformado la muestra definitiva se compone en un $80.6 \%$ de hombres y un $19.4 \%$ de mujeres. El 64\% tiene entre 18 y 22 años. Ha influido en esta distribución la obligación de obtener el consentimiento de los padres de los menores de edad para participar en este estudio, no así entre los mayores de edad. El 76.7\% de los jóvenes es español $(n=120)$. El fracaso escolar es generalizado: un $60.4 \%$ no cuenta con el graduado escolar aun teniendo 16 años o más. Un $12.1 \%$ de los jóvenes de la muestra trabaja. Las medidas que estaban cumpliendo los jóvenes y la reincidencia se muestran en la Tabla 1.

\section{Tabla 1.}

Medidas impuestas y reincidencia.

\begin{tabular}{c}
\hline Internamiento: $6.7 \%$ \\
Libertad vigilada: $70 \%$ \\
\hline Convivencia grupo educativo: $12.7 \%$ \\
\hline Centro de día: $1.3 \%$ \\
\hline Prestación servicios comunidad: $4 \%$ \\
\hline Tareas socioeducativas: $5.3 \%$ \\
\hline REINCIDENCIA: $41.3 \%$
\end{tabular}

\section{Resultados}

La primera de las dimensiones explora la percepción de los menores acerca de las oportunidades que han tenido a lo largo del procedimiento de hablar sobre sus experiencias tras la comisión del delito (Tabla 2): 


\section{Tabla 2.}

Posibilidad de hablar sobre sus experiencias tras la comisión del delito durante el procedimiento penal

\begin{tabular}{l|c|c|c} 
& $\mathrm{SI}$ & $\mathrm{NO}$ & $\mathrm{NS} / \mathrm{NC}$ \\
\hline $\begin{array}{l}\text { Cuando ocurrieron los hechos y hablé con } \\
\text { la policía me preguntaron sobre lo que } \\
\text { había pasado }\end{array}$ & $74.8 \%$ & $20.6 \%$ & $4.5 \%$ \\
\hline $\begin{array}{l}\text { En comisaría pude contar todo lo ocurrido } \\
\text { a un abogado }\end{array}$ & $53.2 \%$ & $39 \%$ & $7.8 \%$ \\
\hline $\begin{array}{l}\text { En comisaría pude contar todo lo ocurrido } \\
\text { a un abogado sin ningún policía presente }\end{array}$ & $40.8 \%$ & $47.4 \%$ & $11.8 \%$ \\
\hline $\begin{array}{l}\text { Cuando llegué al juzgado la primera vez } \\
\text { me preguntaron sobre lo ocurrido }\end{array}$ & $74.5 \%$ & $18.3 \%$ & $7.2 \%$ \\
\hline $\begin{array}{l}\text { Antes del juicio pude hablar con mi } \\
\text { abogado sobre lo ocurrido }\end{array}$ & $81.7 \%$ & $14.4 \%$ & $3.9 \%$ \\
\hline $\begin{array}{l}\text { En algún momento le he podido explicar al } \\
\text { juez mi versión de los hechos }\end{array}$ & $51.6 \%$ & $46.4 \%$ & $3 \%$ \\
\hline $\begin{array}{l}\text { Al empezar a cumplir la medida judicial, } \\
\text { los técnicos o educadores me preguntaron } \\
\text { sobre lo ocurrido }\end{array}$ & $84.2 \%$ & $12.5 \%$ & $3.3 \%$ \\
\hline
\end{tabular}

De este grupo de preguntas se desprende que es muy distinta la percepción que tienen los jóvenes de la posibilidad de comentar sus experiencias con los distintos operadores jurídicos.

Ninguno de ellos alcanza una mayoría cercana a la totalidad, pero los abogados que asisten antes del juicio y los técnicos y educadores responsables de la ejecución de las medidas son, con un $81.7 \%$ y un $84.2 \%$ respectivamente, los profesionales a los que más cantidad de menores cuentan los hechos ocurridos.

Tres de cada cuatro menores sí recuerdan que en comisaría y en fiscalía les preguntaran sobre lo sucedido, pero son muchos los que consideran que no se les pidió ninguna explicación.

Sin duda, la información más llamativa en esta dimensión es la relativa al juez y a los letrados que asisten en comisaría. Respecto al primero, sorprende que la mitad de los condenados no pudieron explicarse o no saben si lo hicieron. Esta constatación se 
puede deber en parte al hecho de que en muchos casos no se celebra la vista oral al llegarse a una conformidad entre las partes. Por tanto, el menor pasa por sede judicial sin haber podido hablar personalmente ante el juzgador de su caso. Sin duda, no todos los casos se pueden explicar con este motivo.

La información respecto a la asistencia de abogado en comisaría es aún más inquietante pues aquí no se encuentra ninguna causa explicativa, ni siquiera parcial, de la información vertida en las respuestas. La mitad de los menores no son conscientes de haber tenido asistencia letrada a su paso por la comisaría y un $26.3 \%$ de aquéllos que recuerdan haber hablado con un abogado en las dependencias policiales lo hizo con un policía también presente.

La segunda dimensión mide la percepción de los menores sobre las posibilidades que han tenido de expresar sus sentimientos en relación a sus experiencias tras la comisión del delito (Tabla 3).

\section{Tabla 3.}

Posibilidad de expresar sus sentimientos respecto a sus experiencias tras la comisión del delito

\begin{tabular}{|c|c|c|c|}
\hline & SI & $\mathrm{NO}$ & $\mathrm{NS} / \mathrm{NC}$ \\
\hline $\begin{array}{l}\text { En las primeras visitas al juzgado hablé con los } \\
\text { profesionales de allí sobre cómo me sentía } \\
\text { acerca de lo sucedido (enfadado, confuso, } \\
\text { triste...) }\end{array}$ & $61.9 \%$ & $32.3 \%$ & $5.8 \%$ \\
\hline $\begin{array}{l}\text { Al abogado le he podido contar cómo me sentía } \\
\text { con todo lo que había pasado }\end{array}$ & $62.7 \%$ & $32 \%$ & $5.2 \%$ \\
\hline $\begin{array}{l}\text { Al empezar a cumplir la medida judicial, hablé } \\
\text { con los técnicos o educadores sobre cómo me } \\
\text { sentía acerca de lo sucedido (enfadado, } \\
\text { confuso, triste...) }\end{array}$ & $81.7 \%$ & $14.4 \%$ & $3.9 \%$ \\
\hline
\end{tabular}

Como era de esperar, la comunicación y expresión de los sentimientos y emociones no ha sido tan usual, especialmente con los operadores con los que los menores se encuentran un número mínimo de ocasiones, como el personal de físcalía y los 
abogados. No obstante, más de la mitad de los jóvenes considera que sí han podido compartir este aspecto de su experiencia.

La percepción de los menores cambia notablemente cuando se les pregunta por sus posibilidades de comunicación con los técnicos y educadores de ejecución: una mayoría de jóvenes comparte con ellos sus emociones. Esta situación está relacionada con el papel educativo que tienen estos profesionales en el procedimiento. Sin embargo, hay un grupo que no lo hace, sin poder dilucidar si la causa está en la decisión del propio menor de no compartir estos aspectos o en causas ajenas a él.

La tercera dimensión se refiere a la percepción del menor acerca de la imparcialidad de las decisiones adoptadas respecto a él durante el procedimiento (Tabla 4).

\section{Tabla 4.}

Percepción del menor sobre la imparcialidad de las decisiones adoptadas durante el procedimiento

\begin{tabular}{l|c|c|c} 
& $\mathrm{SI}$ & $\mathrm{NO}$ & $\mathrm{NS} / \mathrm{NC}$ \\
\hline $\begin{array}{l}\text { Creo que la policía me habría detenido igualmente } \\
\text { aunque fuera de otro barrio }\end{array}$ & $69.9 \%$ & $19.6 \%$ & $10.5 \%$ \\
\hline $\begin{array}{l}\text { Creo que la policía me habría detenido igualmente } \\
\text { me conociera o no de antes }\end{array}$ & $72.9 \%$ & $18.7 \%$ & $8.4 \%$ \\
\hline $\begin{array}{l}\text { Creo que la policía me habría detenido igualmente } \\
\text { aunque mi imagen o forma de vestir fueran } \\
\text { diferentes }\end{array}$ & $63 \%$ & $26.6 \%$ & $10.4 \%$ \\
\hline $\begin{array}{l}\text { Creo que la policía me habría detenido igualmente } \\
\text { aunque fuera de otra raza o nacionalidad }\end{array}$ & $72.7 \%$ & $18.8 \%$ & $8.4 \%$ \\
\hline $\begin{array}{l}\text { Creo que el juez me habría puesto la misma } \\
\text { medida me conociera o no de antes }\end{array}$ & $61.2 \%$ & $25.7 \%$ & $13.2 \%$ \\
\hline $\begin{array}{l}\text { Creo que el juez me habría puesto la misma } \\
\text { medida aunque mi imagen o forma de vestir fueran } \\
\text { diferentes }\end{array}$ & $63.4 \%$ & $21.6 \%$ & $15 \%$ \\
\hline $\begin{array}{l}\text { Creo que el juez me habría puesto la misma } \\
\text { medida aunque fuera de otra raza o nacionalidad }\end{array}$ & $69.7 \%$ & $16.4 \%$ & $13.8 \%$ \\
\hline
\end{tabular}


La percepción relativa a la imparcialidad de los operadores jurídicos no está tan ampliamente extendida entre los condenados como otros aspectos. Se podría decir que aquí se constata la sinceridad de las respuestas de los jóvenes encuestados: es en esta dimensión donde hay un mayor nivel de "NS/NC"; los menores no hacen una valoración de un tema que no conocen.

En esta dimensión resultan muy interesantes las respuestas referidas a las actuaciones policiales. La imagen y/o la forma de vestir es el aspecto que más menores considera que distorsiona la imparcialidad de la policía, pero también vivir en un barrio determinado, ser conocido con anterioridad por estos profesionales y tener otra raza o nacionalidad son circunstancias influyentes.

Es digno de ser destacado que la opinión de los que piensan que el barrio en el que se reside marca una diferencia en la actuación de la policía se diferencia en atención a la edad de los menores. A mayor edad más común es la opinión de la falta de imparcialidad, siendo las diferencias estadísticamente significativas entre los menores de 18 y los que tienen entre 19 y 24 años $\left(\chi^{2}=6,387\right.$; g.l. $\left.=2 ; p=0,04\right)$. Por otra parte, el haber sido condenado una o más veces es también un elemento relacionado con la opinión sobre la actuación policial y su imparcialidad. Los menores que han pasado más de una vez por el procedimiento judicial consideran más a menudo que las actuaciones policiales se ven afectadas por tener anteriores contactos policiales $\left(\chi^{2}=10,316\right.$; g.1. $\left.=4 ; p=0,006\right)$, su forma de vestir $\left(\chi^{2}=5,868 ;\right.$ g.l. $\left.=2 ; p=0,05\right)$ y su raza o nacionalidad $\left(\chi^{2}=9,121 ;\right.$ g.l. $\left.=2 ; p=0,01\right)$ que aquellos que es la primera vez que se encuentran en esta situación.

Si se centra ahora la atención en la imparcialidad judicial percibida por los menores, se observa que la opinión más generalizada es que el ser ya conocido por estos operadores es lo que más la distorsiona, seguida de cerca por la imagen del joven o su forma de vestir. Son menos los que consideran que la raza o nacionalidad influye en este aspecto de la justicia. Aquí también se aprecian diferencias significativas entre grupos. Así, los hombres son más proclives a pensar que ser conocido por el juez tiene influencia en las decisiones que éste toma y también lo son a no contestar a la pregunta $\left(\chi^{2}=7,952\right.$; g.l. $=2 ; p=0,01)$. La edad es también un elemento diferenciador, en este caso en relación a la influencia de la imagen o forma de vestir en la aplicación de la justicia. Aquí son los 
más jóvenes los que consideran que esta circunstancia afectó al juez en su decisión $\left(\chi^{2}=8,606 ;\right.$ g.l. $\left.=2 ; p=0,01\right)$.

La cuarta dimensión explora la percepción del menor sobre la legalidad de las decisiones adoptadas durante el procedimiento que le atañe (Tabla 5).

\section{Tabla 5.}

Percepción del menor sobre la legalidad de las decisiones adoptadas durante el procedimiento

\begin{tabular}{l|c|c|c} 
& $\mathrm{SI}$ & $\mathrm{NO}$ & $\mathrm{NS} / \mathrm{NC}$ \\
\hline $\begin{array}{l}\text { Desde el primer momento creo que la } \\
\text { policía actuó respetando las leyes }\end{array}$ & $49.7 \%$ & $43.2 \%$ & $7.1 \%$ \\
\hline $\begin{array}{l}\text { Creo que mi abogado actúa respetando } \\
\text { las leyes }\end{array}$ & $85.4 \%$ & $7.3 \%$ & $7.3 \%$ \\
\hline $\begin{array}{l}\text { Creo que el juez actuó respetando las } \\
\text { leyes }\end{array}$ & $80.3 \%$ & $10.4 \%$ & $9.3 \%$
\end{tabular}

La percepción de legalidad es diferente según el operador al que se alude. La mayoría de los jóvenes tienen buena opinión en este aspecto en relación a su abogado y también la mayoría piensan así de la actuación de los jueces. Esta última opinión se relaciona con la nacionalidad, sólo algo más de la mitad los extranjeros perciben la legalidad en las decisiones judiciales y el $26.5 \%$ desconocen o no contestan a esta pregunta, lo cual puede ser lógico atendiendo a sus circunstancias de incorporación a la cultura y sistema legal españoles $\left(\chi^{2}=14,742 ;\right.$ g.l. $\left.=2 ; \mathrm{p}=0,001\right)$. Sin embargo, es inquietante la percepción de casi la mitad de la muestra respecto a la legalidad de la actuación de la policía al ser unos niveles notablemente altos y compartidos de desconfianza. Además, esta opinión está relacionada con haber sido condenado antes o no, de manera que los menores reincidentes opinan más a menudo que la policía no respeta las leyes $\left(\chi^{2}=14,863 ;\right.$ g.l. $\left.=2 ; p=0,001\right)$.

La quinta dimensión se centra en la percepción del menor acerca del trato que recibe durante el procedimiento (Tabla 6). 
Tabla 6.

Percepción del menor acerca del trato recibido durante el procedimiento

\begin{tabular}{l|c|c|c} 
& $\mathrm{SÍ}$ & $\mathrm{NO}$ & $\mathrm{NS} / \mathrm{NC}$ \\
\hline La policía me trató con educación y respeto & $52.6 \%$ & $44.2 \%$ & $3.2 \%$ \\
\hline $\begin{array}{l}\text { Los profesionales del juzgado me prestaron atención } \\
\text { cuando hablé con ellos }\end{array}$ & $75.7 \%$ & $17.9 \%$ & $6.4 \%$ \\
\hline $\begin{array}{l}\text { Los profesionales del juzgado me trataron con } \\
\text { educación y respeto }\end{array}$ & $82.4 \%$ & $9.8 \%$ & $7.8 \%$ \\
\hline El abogado me presta atención cuando hablo con él & $83 \%$ & $9.2 \%$ & $7.8 \%$ \\
\hline El abogado me trata con educación y respeto & $92.8 \%$ & $3.9 \%$ & $3.3 \%$ \\
\hline El juez me presta atención cuando hablo con él & $71.7 \%$ & $17.8 \%$ & $10.5 \%$ \\
\hline El juez me trata con educación y respeto & $88.8 \%$ & $13 \%$ & $2.6 \%$ \\
\hline $\begin{array}{l}\text { Normalmente, a lo largo de la medida los técnicos y } \\
\text { educadores me prestan atención cuando hablo con ellos }\end{array}$ & $94.7 \%$ & $4 \%$ & $1.3 \%$ \\
\hline $\begin{array}{l}\text { Normalmente, a lo largo de la medida los técnicos y } \\
\text { educadores me tratan con educación y respeto }\end{array}$ & $92.8 \%$ & $3.3 \%$ & $3.9 \%$
\end{tabular}

Esta dimensión se divide en dos tipos de cuestiones: por un lado, si el joven considera que se le ha tratado con educación y respeto y, por otro, si el profesional correspondiente le ha prestado atención.

En cuanto al primer asunto, casi la totalidad de los jóvenes considera que el abogado y los técnicos de ejecución les tratan con educación y respeto. Esta opinión no está tan generalizada respecto al juez y, aún menos, respecto a los profesionales del juzgado, pero también es muy usual. La peor valoración, sin embargo, es la que hacen de la policía, siendo sólo la mitad de los jóvenes los que consideran que el trato ha sido adecuado. En este ámbito, la variable de reincidencia está relacionada tanto con la opinión que tienen los jóvenes del trato recibido por parte de la policía $\left(\chi^{2}=6,252 ;\right.$ g.l. $\left.=2 ; p=0,04\right)$ como del recibido por parte del juez $\left(\chi^{2}=23,420\right.$; g.l. $=2$; $\left.p=0,000\right)$, por tanto las experiencias previas de los jóvenes con la justicia penal marcan una diferencia.

En el segundo aspecto de esta dimensión, relacionado con la atención prestada a los menores, la opinión más unánimemente favorable es la referida a los técnicos y educadores. Aunque con algo menos de consenso, también la mayoría de los jóvenes

Revista Española de Investigación Criminológica

Artículo 9, Número 16 (2018)

https://doi.org/10.46381/reic.v16i0.171

www.criminologia.net

ISSN: 1696-9219 
considera que su abogado les escucha con atención pero la situación cambia con los operadores de las oficinas judiciales: tres de cada cuatro menores considera que el personal de fiscalía y también el juez les prestaron atención.

La circunstancia personal de los menores que revela diferencias significativas en el caso de las distintas opiniones respecto al trato y atención de los profesionales es la edad. Son los menores de 18 los que perciben más a menudo que la policía y el juez les tratan educadamente $\left(\chi^{2}=6,328 ;\right.$ g.1. $=2 ; p=0,04$ y $\chi^{2}=6,725 ;$ g.1. $\left.=2 ; p=0,03\right)$.

La sexta dimensión pretende medir el grado de comprensión que posee el menor sobre el fundamento general del procedimiento y de las decisiones que se toman a lo largo del mismo (Tabla 7). 


\section{Tabla 7.}

Grado de comprensión por parte del menor del fundamento general del procedimiento y de las decisiones que se toman a lo largo del mismo

\begin{tabular}{|c|c|c|c|}
\hline & Sí & NO & $\mathrm{NS} / \mathrm{NC}$ \\
\hline Comprendo por qué me detuvo la policía & $86.3 \%$ & $7.8 \%$ & $5.9 \%$ \\
\hline $\begin{array}{l}\text { Desde mi primera visita al juzgado supe que el juez } \\
\text { podía imponerme una medida }\end{array}$ & $78.7 \%$ & $14.2 \%$ & $7.1 \%$ \\
\hline $\begin{array}{l}\text { En el juzgado me explicaron que a la hora de imponerme } \\
\text { el juez una medida, también se tendrían en cuenta mis } \\
\text { circunstancias personales y familiares }\end{array}$ & $54.8 \%$ & $37.5 \%$ & $7.7 \%$ \\
\hline $\begin{array}{l}\text { El fiscal tuvo en cuenta el delito que había cometido a } \\
\text { la hora de pedir la medida judicial }\end{array}$ & $71.6 \%$ & $11 \%$ & $17.4 \%$ \\
\hline $\begin{array}{l}\text { El fiscal tuvo en cuenta mis circunstancias personales y } \\
\text { familiares a la hora de pedir la medida judicial }\end{array}$ & $42.6 \%$ & $35.5 \%$ & $21.9 \%$ \\
\hline El papel del abogado es defender a los menores & $80.9 \%$ & $8.6 \%$ & $10.5 \%$ \\
\hline $\begin{array}{l}\text { El juez tuvo en cuenta el delito que cometí a la hora de } \\
\text { imponerme la medida }\end{array}$ & $77 \%$ & $9.9 \%$ & $13.2 \%$ \\
\hline $\begin{array}{l}\text { El juez tuvo en cuenta mis circunstancias personales y } \\
\text { familiares a la hora de imponerme la medida }\end{array}$ & $52.9 \%$ & $26.8 \%$ & $20.3 \%$ \\
\hline $\begin{array}{l}\text { Desde el inicio del cumplimiento de mi medida, me han } \\
\text { explicado que las medidas judiciales, además de } \\
\text { "castigarme", pretenden que yo cambie }\end{array}$ & $86.9 \%$ & $10.5 \%$ & $2.6 \%$ \\
\hline $\begin{array}{l}\text { Me parece bien que, aunque haya cometido el mismo } \\
\text { delito que otro chico, me pongan una medida distinta a } \\
\text { la suya porque mis circunstancias personales son } \\
\text { diferentes }\end{array}$ & $50.7 \%$ & $38 \%$ & $11.3 \%$ \\
\hline $\begin{array}{l}\text { La medida que estoy cumpliendo me está sirviendo para } \\
\text { algo }\end{array}$ & $81.5 \%$ & $13.9 \%$ & $4.6 \%$ \\
\hline $\begin{array}{l}\text { Durante la ejecución de la medida los técnicos o } \\
\text { educadores valoran mi comportamiento para informar al } \\
\text { juez }\end{array}$ & $96.1 \%$ & $2 \%$ & $2 \%$ \\
\hline $\begin{array}{l}\text { El papel de técnicos y educadores es educarme para que } \\
\text { mejore }\end{array}$ & $92.1 \%$ & $3.3 \%$ & $4.6 \%$ \\
\hline
\end{tabular}

Se desprende de las respuestas dadas que, en general, los menores comprenden los fundamentos básicos del procedimiento penal en el que están encartados. La actuación de la policía al intervenir tras la comisión de un ilícito penal, la participación de un abogado que defienda su posición en el procedimiento iniciado, la respuesta de la 
sociedad por medio del juez al imponer una medida sancionadora y el conocimiento de que el "castigo" tiene asociado un objetivo rehabilitador son elementos esenciales que los jóvenes condenados conocen. Todos estos aspectos son compartidos por el procedimiento penal de adultos, por lo que podríamos decir que este grupo de población tiene una socialización legal básica adecuada.

Por su parte, el contenido educativo de la justicia de menores no es explícito para la mayoría de ellos, a pesar de ser los "usuarios" directos de la misma. Sólo la mitad reconoce o recuerda que se le explicó tal aspecto y uno de cada tres niega conocerlo. Prácticamente la misma distribución se reproduce en la respuesta a la pregunta de si les parece bien que las medidas impuestas sean distintas en el caso de que el ilícito cometido sea similar al de otro chico porque las circunstancias personales cambien. Es decir, la mitad de los jóvenes condenados no fue consciente al inicio del procedimiento de este objetivo legal, y además, muchos de ellos ni siquiera parecen estar de acuerdo con el mismo.

Otra faceta de este mismo asunto es la información relativa a los criterios que han tenido en cuenta el fiscal y el juez al proponer e imponer la sanción. Tres de cada cuatro chicos afirman que el delito fue tenido en cuenta, lo cual es lógico en esa socialización legal básica que antes se refirió. Sin embargo, sólo la mitad reconoce la consideración en su caso de las circunstancias personales por parte del juez y algo inferior es el porcentaje de condenados que afirma que se tuvieron presentes por parte del fiscal. Aquí también la información que proporcionan las respuestas negativas y las de los que no saben o no contestan es reveladora. El $26.8 \%$ afirma que el juzgador no tuvo en cuenta sus circunstancias personales y aumenta hasta un 35.5 el porcentaje de los que perciben que el fiscal tampoco contó con ellas. Nótese también que uno de cada cuatro encuestados simplemente desconoce o no contesta a esta cuestión, siendo las respuestas con más alto porcentaje en esta dimensión.

Por último, prácticamente la totalidad de los encuestados hace patente que la intervención del control social formal tras la comisión de su infracción tiene una consecuencia útil en su vida y que el interés último que tiene el trabajo realizado por los técnicos de ejecución es educativo. Por tanto, la intención rehabilitadora, resocializadora 
y educativa de la justicia juvenil se aprecia claramente por parte de los jóvenes en este periodo final del proceso.

Un último apunte a destacar es que ninguna de estas preguntas guarda relación con el hecho de haber sido condenado una o más veces, con lo que se aprecia que esta circunstancia no aporta más comprensión al menor sobre el procedimiento penal en el que participa.

\section{Discusión}

Este estudio desgrana la percepción del proceso penal como justo por parte de menores sancionados atendiendo a los cuatro elementos aportados por la justicia procedimental y en relación a cada uno de los operadores jurídicos. Se identifican notables diferencias en las experiencias vividas con éstos, pero todos contribuyen a la percepción del joven de haber sido tratado de una forma justa por el sistema penal, lo que tendrá connotaciones en su posterior comportamiento delictivo (Bouffard \& Piquero, 2010)

A este respecto, el operador jurídico sobre el que se tiene una percepción más negativa es la policía. Este resultado es acorde al recientemente obtenido por Baz y Fernández-Molina (2017) entre población adolescente española, según el cual la peor evaluación sobre la policía es emitida por aquellos jóvenes que han tenido una relación directa con ella. Resultados similares alcanzan estudios realizados en diferentes países, Australia (Hinds, 2007), Gran Bretaña (Bradford, 2012), Nigeria (Akinlabi, 2015), Finlandia (Saarikkomäki, 2016), EE. UU. (Vidal et al., 2017). Se revela que, tanto la ausencia de un trato justo por su parte, como la ilegalidad de sus actuaciones, son una opinión habitual entre estos jóvenes; no obstante suelen comprender el porqué de su detención y tener una cierta percepción de imparcialidad de la misma. Esta distinción deja ver que es la interacción personal la que más afecta de forma negativa a la relación entre los menores y la policía: no aprecian una comunicación educada, respetuosa y atenta por parte de los agentes. En el mismo sentido, la reciente investigación llevada a cabo por Tyler (2017) mostró que los jóvenes que comprendían la legitimidad de la acción policial se sintieron tratados de un modo más justo que el resto, siendo el predictor más determinante de la falta de justicia procedimental el lenguaje duro e insultante de estos 
agentes. Hay que destacar que normalmente la "primera cara" del sistema de justicia penal que ven los adolescentes es la de la policía y su confianza en el mismo se genera por el trato recibido. Consecuentemente, estos encuentros tienen mucha relevancia en la consideración de la legitimidad de las autoridades que los jóvenes van construyendo en su proceso de socialización (Fagan \& Tyler, 2005; Gau \& Brunson, 2010; Tyler, 2015; Tyler, Fagan, \& Geller, 2014).

Los letrados son percibidos por los menores como profesionales que actúan de acuerdo a la legalidad y con los que pueden hablar: sienten la atención que les prestan y el trato respetuoso y educado que reciben, como se deja ver en otras investigaciones (Chui \& Cheng, 2017; Sprott \& Greene, 2008) y estas experiencias están conectadas a la percepción de legitimidad del sistema de justicia (Chui \& Cheng, 2015). Sin embargo, sorprende la escasa importancia que se le da a la asistencia letrada inmediata de los menores, ya en comisaria, y aún más a la comunicación privada con un abogado desde el momento en que son objeto de una acusación; sin duda estas circunstancias están relacionadas con el "rol complicado" que mantienen en la justicia juvenil (FernándezMolina, 2013).

Los profesionales que trabajan en la fiscalía de menores, entre los que se incluye el equipo técnico responsable de realizar el estudio psicosocial, consiguen una comunicación adecuada con los infractores que llegan a sus dependencias: los menores sienten en su mayoría un trato en el que hay educación y respeto, pueden hablar sobre lo ocurrido y estiman que se les presta atención, aunque son menos los que perciben que hay posibilidad de comunicar sus sentimientos. La incidencia de esto contactos en la confianza hacia la justicia, de la calidad del trato y de las decisiones adoptadas por los fiscales se ha confirmado en algunas investigaciones (Greene et al., 2010; Sprott \& Greene, 2008)

Respecto al papel de los jueces de menores, ya se resaltó anteriormente el hecho de que pocos jóvenes considerasen que podían explicarle al juez su versión de los hechos en algún momento del procedimiento. Al cruzar las opiniones de este grupo y de los que afirman que este profesional les trató con educación y respeto y prestándoles atención, se puede conocer más en detalle esta situación. Así, sólo un 3.8\% de los que hablaron de su

Artículo 9, Número 16 (2018)

https://doi.org/10.46381/reic.v16i0.171

www.criminologia.net

ISSN: 1696-9219 
caso con el juez sienten que no les prestó atención y el $2.5 \%$ que no hubo atención ni respeto, lo cual es un dato muy positivo y coincidente con diversas investigaciones (Bornstein et al., 2016; Greene et al., 2010). No obstante, si los jóvenes no pueden hablar con el juez sobre lo sucedido se marca una diferencia en su percepción de un comportamiento adecuado del juez ${ }^{2}$ (Sprott \& Greene, 2008), aunque sean las normas procesales las que justifiquen esta situación.

Por su parte y sin ninguna duda, los profesionales que más contribuyen a la consideración de un trato justo por el sistema de justicia juvenil son los técnicos y educadores encargados de gestionar la ejecución de las medidas sancionadoras. Así, a pesar de que la relación entre el profesional y el infractor es prolongada y no se dirime en uno o dos actos procesales de escasa duración, en torno al 92\% de ellos considera que son tratados con educación y respeto y comprenden la labor educativa de que son objeto. También una amplia mayoría, más del $80 \%$, considera que hablan sobre lo ocurrido y comparten sus sentimientos con estos profesionales. Estas respuestas confirman la excelente labor profesional que llevan a cabo estos especialistas, influyente en la opinión sobre la legitimidad de la justicia (Steiner \& Wooldredge, 2015) y que no es mediatizada por las percepciones negativas que han podido tener de los demás operadores jurídicos, como sucede en otros casos (Tatar et al., 2012).

\section{Limitaciones del estudio}

La primera limitación está relacionada con consideraciones teóricas: en las preguntas sobre la calidad del trato se ha dado un amplio protagonismo a las relativas a la percepción de elementos particulares de nuestra justicia juvenil, aspecto no muy contrastado en la literatura internacional. Además, aunque la muestra de jóvenes condenados fue seleccionada de forma aleatoria, hay una representación más amplia de jóvenes mayores de edad por las razones arriba apuntadas, lo cual es un sesgo de la misma. El instrumento de medida ha sido conformado intentando garantizar la claridad del contenido y la fácil

\footnotetext{
${ }^{2}$ Las diferencias son significativas cuando se pregunta si el juez les prestó atención $\left(\chi^{2}=26,663\right.$; g.l. $=4$; $\mathrm{p}=0,000)$ y si les trata con educación y respeto $\left(\chi^{2}=10,310 ;\right.$ g.l. $\left.=4 ; \mathrm{p}=0,03\right)$.
} 
comprensión de los términos utilizados por los jóvenes, cerrando las respuestas entre "sí", "no" y "no sabe/no contesta" lo cual ha supuesto renunciar a la riqueza que hubiera aportado la utilización de una escala Likert. Tampoco se han incluido preguntas relativas a la percepción de la legitimidad como suele ser habitual en este tipo de estudios, de modo que no se ha podido correlacionar en qué medida la percepción de un trato justo en el procedimiento influye en la opinión sobre la legitimidad del sistema penal juvenil

\section{Conclusiones}

Este estudio piloto aporta indicios sobre la ausencia de características de la justicia procedimental que hacen percibir el proceso penal juvenil como justo a los jóvenes infractores. Además, las diferencias en la valoración de los distintos profesionales que contactan con ellos a lo largo del procedimiento parecen ser notables. Se ha mostrado en las diversas investigaciones revisadas la importancia que tiene para los infractores la percepción de ser tratados de un modo justo por las autoridades penales: no sólo influye en su percepción general de legitimidad de la autoridad, sino que esta opinión se mantiene en el tiempo y está relacionada con el mantenimiento del comportamiento delictivo, sobre todo entre los jóvenes adultos. Los resultados hallados apuntarían a que la percepción de ser objeto de un proceso justo de los jóvenes sancionados puede ser mejorada a partir de dos aspectos principales: 1) la policía ha de ser consciente de que el trato que da a los jóvenes a la hora de ser imputados o detenidos es un elemento que no es neutral para el posterior comportamiento delictivo del menor; 2) la explicación al menor de lo que sucede y por qué sucede ha de incorporarse en las actuaciones de casi todos los operadores jurídicos, algo que influirá en que acepte las consecuencias del proceso penal y tenga una actitud más positiva ante las decisiones judiciales. Este estudio piloto confirma el potencial explicativo del examen diferenciado de cada uno de los momentos procesales existentes, así como el análisis de su peso singularizado en la percepción global del proceso penal juvenil como justo. El conocimiento de estos aspectos posibilitará plantear propuestas efectivas que incidan en el cumplimiento normativo de los jóvenes infractores. 


\section{Referencias}

Akinlabi, O. (2015). Young people, procedural justice and police legitimacy in Nigeria. Policing and Society, 27, 419-438. DOI 10.1080/10439463.2015.1077836

Agustyn, M. B. (2016). Updating perception of (in)justice. Journal of Research in Crime and Delinquency, 53, 255-286.

\section{DOI 10.1177/0022427815616991}

Baz, O. \& Fernández-Molina, E. (2017). Process-based model in adolescent. Analyzing police legitimacy and juvenile delinquency within a legal socialization framework. European Journal on Criminal Policy and Research, 1-16.

DOI 10.1007/s10610-017-9357-y

Bernuz Beneitez, M. J. (2014). La legitimidad de la justicia de menores: entre justicia procedimental y justicia social. InDret 1/14.

Birckhead, T. (2009). Toward a theory of procedural justice for juveniles. Buffalo Law Review, 57, 1447-1513.

Blader, S. \& Tyler, T. (2003). A four-component model of procedural justice: Defining the meaning of a "fair" process. Personality and Social Psychology Bulletin, 29, 747-758. DOI 10.1177/0146167203252811

Boonie, R., Johnson, R., Chemers, R., \& Schuck, J. (2013). Reforming Juvenile Justice. A Developmental Approach. Washington, D.C.: The National Academies Press.

Bornstein, A., Marcus, A., Curtis, R., Rivera, S., \& Swaner, R. (2016). Tell it to de Judge: procedural justice and a community court in Brooklyn. Political and legal Anthropology Review, 39, 206-225. DOI 10.1111/plar.12190

Bouffard, L. A. \& Piquero, N. L. (2010). Defiance theory and life course explanations of persistent offending. Crime and Delinquency, 56, 227-252. DOI $10.1177 / 0011128707311642$

Bradford, B. (2014). Policing and social identity: procedural justice, inclusion and cooperation between police and public. Policing and Society, 24, 22-43. DOI $10.1080 / 10439463.2012 .724068$

Braga, A. A. \& Weisburd, D. L. (2012). The effects of focused deterrence strategies on crime: A systematic review and meta-analysis of the empirical evidence. Journal of research in crime and delinquency, 49, 323-358.

DOI $10.1177 / 0022427811419368$

Chui, W. H. \& Cheng, K. (2015). Young people's perception of lawyers in Hong Kong: A comparison between offenders, youth-at-risk and students. International Journal of Law, Crime and Justice, 43, 481-495. DOI 10.1016/j.ijlcj.2014.12.001

Chui, W.H. \& Cheng, K. (2017). Perceptions of Fairness and Satisfaction in LawyerClient Interactions Among Young Offenders in Hong Kong. Journal of mixed methods research, 11, 266-285. DOI 10.1177/1558689815593834

Revista Española de Investigación Criminológica

Artículo 9, Número 16 (2018)

https://doi.org/10.46381/reic.v16i0.171

www.criminologia.net

ISSN: 1696-9219 
Cohn, E., Trinkner, R., Rebellon, C., Van Gundy, K. \& Cole, L. (2012). Legal Attitudes and Legitimacy: Extending the Integrated Legal Socialization Model. Victims and offenders, 7, 385-406. DOI 10.1080/15564886.2012.713902

Dai, M., Frank, S. \& Sun, I. (2011). Procedural justice during police-citizen encounters: The effects of process-based policing on citizen compliance and demeanor. Journal of Criminal Justice, 39, 159-168. DOI 10.1016/j.jcrimjus.2011.01.004

Fagan, J. \& Piquero, A. (2007). Rational Choice and Developmental Influences on Recidivism Among Adolescent Felony Offenders. Journal of Empirical Legal Studies. 4, 715-748. DOI 10.1111/j.1740-1461.2007.00105.x

Fagan, J. \& Tyler, T. (2005). Legal socialization of Children and Adolescents. Social Justice Research, 18, 217-241.

DOI $10.1007 / \mathrm{s} 11211-005-6823-3$

Fernández-Molina, E. (2013). Una aproximación a la figura del abogado en la justicia de menores. Cuadernos de Política criminal, 109, 217-242.

Gau, J. M. \& Brunson, R. K. (2010). Procedural Justice an Order Maintenance Policing: A Study of Inner-City Young Men's Perceptions of Legitimacy. Justice Quartterly, 27, 163-194. DOI 10.1080/07418820902763889

Greene, C., Sprott, J. B., Madon, N. S., \& Jung, M. (2010). Punishing processes in youth court: Procedural justice, Court Atmosphere ans Youths' views of the legitimacy of the justice system. Canadian journal of Criminology and Criminal justice, 52, 527-544. DOI 10.3138/cjecj.52.5.527

Hinds, L. (2007). Building Police-Youth Relationships: The Importance of Procedural Justice. Youth Justice, 7,195-209. DOI 10.1177/1473225407082510

Jackson, J., Bradford, B., Stanko, B., \& Hohl, K. (2012). Just authority? Trust in the police in England and Wales. Routledge, Londres.

Jackson, J., Bradford, B., Hough, M., Myhill, A., Quinton, P., \& Tyler, T. (2012). Why do people comply with the law. Legitimacy and the influence of legal institutions. British Journal of Criminology, 52, 1051-1071. DOI 10.1093/bjc/azs032

Jonathan-Zamir, T., Mastrofski, S. D., \& Moyal, S. (2015). Measuring procedural justice in police-citizen encounters. Justice Quarterly, 32, 845-871.

DOI 10.1080/07418825.2013.845677

Kirk, D.S. \& Matsuda, M. (2011). Legal cynicism, collective efficacy and ecology of arrest. Criminology: an interdisciplinary Journal, 49, 443-472.

DOI 10.1111/j.1745-9125.2011.00226.x

McCluskey, J. D. (2003). Police request for compliance: Coercive and procedurally just tactics. New York: LFB Scholary Publisching.

Revista Española de Investigación Criminológica

Artículo 9, Número 16 (2018)

https://doi.org/10.46381/reic.v16i0.171

www.criminologia.net

ISSN: 1696-9219 
McGarrell, E., Corsaro, N., Hipple, N., \& Bynum, T. (2010). Project safe neighborhoods and violent crime trends in US cities: Assessing violent crime impact. Journal of Quantitative Criminology, 26, 165-190. DOI 10.1007/s10940-010-9091-9

Mulvey, E., Steinberg, L., Fagan, J., Cauffman, E., Piquero, A., Chassin, L.; Knight, G., Brame, R., Shubert, C., Hecker, T. \& Losoya, S. (2004). Theory and Research on Desistance from Antisocial Activity among Serious Adolescent Offenders. Youth Violence and Juvenile Justice, 2, 213-236. DOI 10.1177/1541204004265864

Murphy, K. \& Gaylor, A. (2010). Policing Youth: Can procedural justice nurture youth cooperation with pólice? Alfred Deakin Research Institute, Working paper $n^{0} 6$. Deakin University, Geelong, Australia.

Otto, K. \& Dalbert, C. (2005). Belief in a just world and its functions for young prisioners. Journal or Research in Personality, 39, 559-573. DOI 10.1016/j.jrp.2005.01.004

Penner, E. K., Viljoen, J. L., Douglas, K. S., \& Roesh, R. (2014). Procedural justice versus risk factors for offending: Predicting recidivism in youth. Law and Human

Behavior, 38, 225-237. DOI 10.1037/lhb0000055

Piquero, A., Fagan, J., Mulvey, E., Steinberg, L., \& Odgers, C. (2005). Developmental trajectories of legal socialization among serious adolescent offenders. Journal of Criminal Law and Criminology, 96, 267-298.

Reisig, M. Tankebe, J., \& Mesko, G. (2014). Compliance with the law in Slovenia: The role of procedural justice and police legitimacy._European Journal on Criminal Policy and Research, 20, 259-276. DOI 10.1007/s10610-013-9211-9

Saarikkomäki, E. (2016). Perceptions of procedural justice among young people: narratives of fair treatment in young people's stories of police and security guard intervention. British Journal of Criminology, 56, 1253-1271. DOI 10.1093/bjc/azv102

Schulhofer, S. J., Tyler, T. R., \& Huq, A. (2011). American policing at a crossroads: Unsustainable policies and the procedural justice alternative. The Journal of Criminal Law \& Criminology, 101, 335-374. DOI 0091-4169/11/10102-0335

Sprott, J. \& Greene, C. (2010). Trust and confidence in the courts: Does the quality of treatment young offenders receive affect their views of the courts?. Crime and Delinquency, 56, 269-289. DOI 10.1177/0011128707308176

Steiner, B. \& Wooldredge, J. (2015). Examining the sources of correctional officer legitimacy. Journal of Criminal Law and Criminology, 105, 679-704. DOI 00914169/15/10503-0679

Sunshine, J. \& Tyler, T. (2003). The role of procedural justice and legitimacy in shaping public support for policing. Law and Society Review, 37, 513-547. DOI $10.1111 / 1540-5893.3703002$

Revista Española de Investigación Criminológica

Artículo 9, Número 16 (2018)

https://doi.org/10.46381/reic.v16i0.171

www.criminologia.net

ISSN: 1696-9219 
Tankebe, J. (2013). Viewing things differently: The dimensions of public perceptions of police legitimacy. Criminology, 51, 103-135. DOI 10.1111/j.17459125.2012.00291.x

Tatar, J., Kaasa, S., \& Cauffman, E. (2012). Perceptions of procedural justice among female offenders: Time does not heal all wounds. Psychology, Public Policy and Law, 18, 268-296. DOI 10.1177/0093854813497479

Tyler, T. (1990). Why people obey the law. Yale, University Press, New Haven.

Tyler, T. (2001). Public trust and confidence in legal authorities: What do majority and minority group members want from the law and legal institutions? Behavioral Sciences and the Law, 19, 215-35. DOI 10.1002/bsl.438

Tyler, T. (2003). Procedural justice, legitimacy and the effective rule of the law. En M. Tonry (ed.) Crime and justice: A review of the research, 30, 283-357. Chicago: University of Chicago Press.

Tyler, T. (2009). Legitimacy and criminal justice: The benefits of self-regulation. Ohio State Journal of Criminal Law, 7, 307-359.

Tyler, T. (2015). Why trust matters with juveniles. American Journal of Orthopsychiatry, 85, 83-99. DOI 10.1037/ort0000104

Tyler, T. (2017). Can the police enhance their popular legitimacy through their conduct? Using Empirical Research to Inform Law. University Illinois Law Review, 19, 1971-1208.

Tyler, T., Casper, J. D., \& Fisher, B. (1989). Maintaining Allegiance toward Political Authorities: The Role of Prior Attitudes and the Use of Fair Procedure. American Journal of Political Science, 33, 629-652. DOI 10.2307/2111066

Tyler, T. \& Fagan, J. (2008). Legitimacy and cooperation: Why do people help the police fight crime in their communities? Ohio State Journal of Criminal Law, 6, 231-75

Tyler, T., Fagan, J., \& Geller, A. (2014). Streets stops and police legitimacy: teachable moments in young urban men's socialization. Journal of empirical legal studies, 11, 751-785. DOI 10.1111/jells.12055

Tyler, T., Goff, P., \& MacCoun, R. (2015). The Impact of Psychological Science on Policing in the United States: Procedural Justice, Legitimacy, and Effective Law Enforcement. Psychological Science in the Public Interest, 16, 75-109. DOI $10.1177 / 1529100615617791$

Tyler, T. \& Huo, Y. (2002). Trust the law: Encouraging public cooperation with the police and courts. New York. Russell Sage Foundation.

Tyler, T., Fagan, J., \& Geller, A. (2014). Streets stop and police legitimacy: Teachable moments in young urban men's legal socialization. Journal of Empirical Legal Studies, 11, 751-785. DOI 10.1111/jels. 12055

Revista Española de Investigación Criminológica

Artículo 9, Número 16 (2018)

https://doi.org/10.46381/reic.v16i0.171

www.criminologia.net

ISSN: 1696-9219 
Tyler, T. \& Jackson, J. (2014). Popular Legitimacy and the Exercise of Legal Authority: Motivating Compliance, Cooperation and Engagement. Psychology, Public Policy and Law, 20, 78-95. DOI 10.1037/a0034514

Vázquez Morales, D. \& Fernández Molina, E. (2013). Confianza en los tribunales penales. Una vía normativa a la cooperación ciudadana con la justicia más allá de la amenaza y la coerción. Revista Electrónica de Ciencia Penal y Criminología, 15, $1-29$.

Vidal, S., Cleary, H., Woolard, J., \& Michel, J. (2017). Adolescents' Legal Socialization: Effects of Interrogation and Miranda Knowledge on Legitimacy, Cynicism, and Procedural Justice en Youth Violence and Juvenile justice, 15, 419-440. DOI $10.1177 / 1541204016651479$

Walters, G. D. (2018). Procedural justice, legitimacy beliefs and moral disengagement in emerging adulthood: Explaining continuity and resistance in the moral model of criminal lifestyle development. Law and Moral Behavior, 42, 37-49. DOI $10.1037 / \mathrm{lhb} 0000266$

\section{Financiación}

Este estudio ha sido realizado en el marco del convenio de investigación firmado en abril de 2014 entre la Consejería de Justicia e interior de la Junta de Andalucía y la Universidad de Málaga, vigente durante dos años.

\section{Agradecimiento}

Nuestro más sincero agradecimiento a Eva Ramos Taillefer y a los profesionales encargados de la ejecución de medidas sancionadoras en Málaga sin cuya colaboración no hubiese sido posible la realización de esta investigación.

Fátima Pérez Jiménez es Profesora contratada doctora del área de Derecho penal de la Universidad de Málaga. Directora del equipo de investigación "Observatorio de la delincuencia" (ODA) del Instituto de Criminología.

https://orcid.org/0000-0002-4853-7996

José Becerra Muñoz es profesor ayudante doctor del área de Derecho penal e investigador del Instituto de Criminología de la Universidad de Málaga. Forma parte del equipo ODA.

https://orcid.org/0000-0001-7392-0199

Araceli Aguilar Conde es Profesora/Tutora en el Grado de Criminología de la UNED en Málaga y miembro del equipo investigador del ODA y del Instituto de Criminología desde 2008.

https://orcid.org/0000-0002-0520-418X

Revista Española de Investigación Criminológica

Artículo 9, Número 16 (2018)

https://doi.org/10.46381/reic.v16i0.171

www.criminologia.net

ISSN: 1696-9219 